\title{
Preliminary Report: Multiple Clusters of Proliferating Cells in Non-dysplastic Corrupted Colonic Crypts Underneath Conventional Adenomas
}

\author{
CARLOS A. RUBIO \\ Gastrointestinal and Liver Research Laboratory, Department of Pathology, \\ Karolinska Institute and University Hospital Stockholm, Stockholm, Sweden
}

\begin{abstract}
Background: The finding was recently reported of clusters of colonic crypts lined with indigenous normal epithelium displaying irregular shapes underneath the adenomatous glands of conventional (tubular or villous) adenomas. These abnormal crypts were named non-dysplastic corrupted colonic crypts (NDCs). This study explored the characteristics of cell proliferation in NDCs present in a cohort of conventional adenomas. Materials and Methods: Sections from six conventional adenomas were challenged with the proliferation marker Ki-67 (MIB1). MIB+ proliferating clusters were regarded as those exhibiting two or more adjoining $M I B+$ cells. Results: A total of 46 (range=1-18) NDCs were found underneath the six conventional adenomas. Out of the 46 NDCs, two exhibited only one proliferative cluster/crypt, 14 NDCs two clusters/crypt, 14 three clusters/crypt and the remaining 16 NDCs more than four distinct clusters/crypt. Conclusion: This preliminary study showed, evidently for the first time, that multiple, apparently haphazardly distributed clusters of proliferating cells are present in NDCs. Since the Ki-67 proliferation marker only labels progenitor daughter cells generated by stem cells, each MIB+ cluster in each NDC must have been produced by a single stem cell. Consequently, individual NDCs may harbor several stem cells, a deduction that is in concert with recent studies showing that in the normal human colon, the number of stem cells per crypt is of the order of five to six, or about 5\% of the cell population of a single crypt.
\end{abstract}

This article is freely accessible online.

Correspondence to: Carlos A. Rubio, MD, Ph.D., Gastrointestinal and Liver Pathology Research Laboratory, Department of Pathology, Karolinska Institute and University Hospital, 17176, Stockholm, Sweden. Tel: +46 851774527, Fax: +46 851774524, e-mail: Carlos.Rubio@ki.se

Key Words: Colon, crypts, stem cells, morphology, proliferation.
The mucosa of the colon is constantly challenged by carcinogenic factors able to generate mucosal foci of mutated dysplastic cells called adenomas, lesions with the propensity to progress to invasive carcinoma. This sequence of events is one of the most intensively studied among human malignancies. For many years, the general view was that the vast majority of colonic carcinomas in humans evolved from conventional (tubular or villous) adenomas via the adenoma-carcinoma pathway (1). In conventional adenomas, the dysplastic glands initially develop at the luminal aspect of the lesion and progress downwards, eventually replacing the normal colorectal crypts below, this mechanism being referred to as top-down replacement (2). More recently, serrated colorectal polyps (hyperplastic polyps, sessile serrated polyps and traditional serrated adenomas) have emerged as an alternative pathway of colonic carcinogenesis in humans $(3,4)$. It has been estimated that about $30 \%$ of all colorectal carcinomas progress via the serrated pathway (5). In a study of all colorectal adenomas registered at the Karolinska University Hospital, Stockholm, Sweden, between 1993 and 2000 $(n=3135), 93 \%$ were found to be conventional adenomas and the remaining $7 \%$ were traditional serrated adenomas (serrated and microtubular) (6). Hyperplastic polyps and sessile serrated polyps were not included in the study evaluation,

From the above, it might be deduced that conventional adenoma continues to be the most commonly reported histological phenotype of colorectal adenoma.

In a previous study, colonic crypts lined with indigenous normal epithelium were found, albeit displaying clusters of irregularly shaped crypts below the adenomatous glands in 255 conventional adenomas (7). These crypts with irregular shapes were named non-dysplastic corrupted colonic crypts (NDCs). In this study, sections from conventional adenomas were evaluated with Ki67 antigen, a reliable protein marker of cycling cells, in order to assess the characteristics of cell proliferation in NDCs underneath conventional adenomas. 


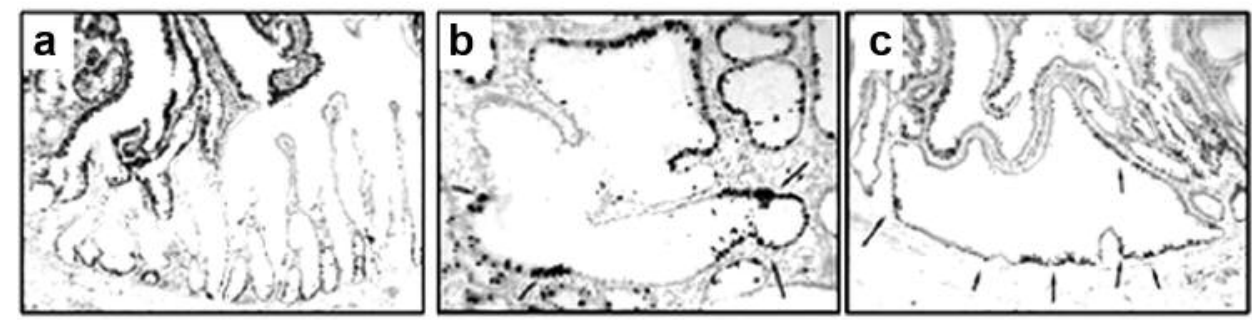

Figure 1. Non-dysplastic corrupted crypts (NDCs) underneath adenomatous glands of conventional colonic adenomas stained for the proliferation marker Ki-67 (clone MIB1). a: Multipartite crypt fission in NDC showing basal cell proliferation. Note irregular adenomatous (dark) glands on top with intense cell proliferation (original magnification, $\times 2$ ). $b$ and $c$ : Detail of two NDCs showing multiple (isolated) clusters of proliferating cells (arrows) (original magnification, $\times 10$ ).

\section{Materials and Methods}

Sections from six conventional adenomas diagnosed at the Department of Pathology, Karolinska University Hospital, were stained with hematoxylin and eosin (H\&E) and immunostained with Ki-67 antigen (clone MIB1; DakoCytomation, Glostrup, Denmark). The monoclonal antibody to Ki-67 detects a nuclear antigen that is present only in proliferating cells. Immunostained sections were scrutinized for MIB1 positive (MIB1+) cells present in the NDC underneath conventional adenomas MIB+ proliferating clusters were regarded as those exhibiting two or more adjoining MIB+ cells. The number of MIB1+ cell clusters within each NDC was recorded.

\section{Results}

A total of 46 (range $=1-18$ ) NDCs were found underneath the six conventional adenomas. Out of the 46 NDCs, two exhibited only one MIB+ cell cluster/crypt, 14 had two MIB+ clusters/crypt, 14 had three and the remaining 16 NDCs had four or more MIB+ clusters/crypt (Figure 1).

\section{Discussion}

The normal mucosa of the colon is built with a single layer of epithelial cells with inward invaginations or folds called crypts. Sections cut perpendicularly to the surface epithelium show a characteristic appearance of 'row of test tubes' with parallel, tightly packed crypts of about the same size, resting on the muscularis mucosae. This architecture is retained throughout the colon, except in nominate grooves (cloverleaf-like crypts connected to a single lumen) (8). Despite the fact that crypts replicate by symmetric fission, beginning at their base and proceeding upwards until two identical individual crypts are created, crypt branching is rarely observed in fixed preparations (9).

Immunostaining for the nuclear antigen KI-67, a protein marker of DNA-synthesizing epithelial cells, reveals that proliferating cells, which are actively committed to cell division, occupy the slopes of the lower fourth of the crypts. In elderly patients (10) and in patients harboring large adenomas or carcinomas elsewhere in the colon $(11,12)$, colonic crypts show upper expansion of the normal proliferative zone. In contrast, in this study, a single zone was not found in NDCs but instead there were multiple (isolated) clusters of proliferating cells.

Fission of colonic crypts is orchestrated by the adenomatous polyposis coli (APC) gene. At the base of the crypt within the stem-cell niche, the stem cells generate more stem cells (13), as well as transient amplifying daughter cells, that is, progenitor cells responsible for the bulk of cell proliferation in the crypt $(14,15)$. Considering that each cluster of proliferating cells is orchestrated by a stem cell (14), the finding of multiple clusters of proliferating cells in NDCs is validated by recent findings indicating that normal colonic crypts are furnished with five to six stem cells (16).

In conclusion, this preliminary study showed, evidently for the first time, that multiple, apparently haphazardly distributed clusters of proliferating cells are present in NDCs. Since the Ki-67 proliferation marker only labels progenitor daughter cells generated by stem cells, each of the multiple MIB+ clusters in each NDC must have been produced by a single stem cell. Consequently, individual NDCs may harbor several stem cells, a deduction that is in concert with recent studies showing that in the normal human colon, the number of stem cells per crypt is of the order of five to six (16), or about $5 \%$ of the cell population of a single crypt (17).

\section{Conflicts of Interest}

The senior Author (KM) provides clinical and academic consultancy services to Q Medical Technologies.

\section{References}

1 Jackman RJ and Mayo CW: The adenoma-carcinoma sequence in cancer of the colon. Surg Gynecol Obstet 93: 327-330, 1951.

2 Shih IM, Wang TL, Traverso G, Romans K, Hamilton SR, BenSasson S, Kinzler KW and Vogelstein B: Top-down morphogenesis of colorectal tumors. Proc Natl Acad Sci USA 98: 2640-2645, 2001. 
3 Longacre TA and Fenoglio-Preiser CM: Mixed hyperplastic adenomatous polyps/serrated adenomas. A distinct form of colorectal neoplasia. Am J Surg Pathol 14: 524-537, 1990.

4 O'Brien MJ, Zhao Q and Yang S: Colorectal serrated pathway cancers and precursors. Histopathology 66: 49-65, 2015.

5 O'Brien MJ and Gibbons D: The adenoma-carcinoma sequence in colorectal neoplasia. Surg Oncol Clin N Am 5: 513-530, 1996.

6 Rubio CA: Colorectal adenomas: Time for reappraisal. Pathol Res Pract 198: 615-620, 2002.

7 Rubio CA and Schmidt PT: Are non-dysplastic corrupted colonic crypts the initial recordable histological event in the development of conventional adenomas? Anticancer Res (submitted).

8 Dahl J and Greenson JK: Colon. In: Histology for Pathologists. Mills SE (eds). Lippincott Williams \& Wilkins. Third edition, Philadelphia, PA, USA Chapter 25, pp. 629-630, 2007.

9 Levine DS, and Haggitt RC: Normal histology of the colon. Am J Surg Pathol 13: 966-984, 1989.

10 Roncucci L, Ponz de Leon M, Scalmati A, Malagoli G, Pratissoli $\mathrm{S}$, Perini $\mathrm{M}$ and Chahin NJ: The influence of age on colonic epithelial cell proliferation. Cancer 62: 2373-2377, 1988.

11 Terpstra OT, van Blankenstein M, Dees $\mathrm{J}$ and Eilers GA: Abnormal pattern of cell proliferation in the entire colonic mucosa of patients with colon adenoma or cancer. Gastroenterology 92: 704-708, 1987.

12 Ponz de Leon M, Roncucci L, Di Donato P, Tassi L, Smerieri O, Amorico MG, Malagoli G, De Maria D, Antonioli A and Chahin NJ: Pattern of epithelial cell proliferation in colorectal mucosa of normal subjects and of patients with adenomatous polyps or cancer of the large bowel. Cancer Res 48: 4121-4126, 1988.
13 Humphries A and Wright NA: Colonic crypt organization and tumorigenesis. Nat Rev Cancer 8: 415-424, 2008.

14 Thirlwell C, Will OC, Domingo E, Graham TA, McDonald SA, Oukrif D, Jeffrey R, Gorman M, Rodriguez-Justo M, ChinAleong J, Clark SK, Novelli MR, Jankowski JA, Wright NA, Tomlinson IP and Leedham SJ: Clonality assessment and clonal ordering of individual neoplastic crypts shows polyclonality of colorectal adenomas. Gastroenterology 138: 1441-1154, 2010.

15 Rubio CA: Putative stem cells in mucosa of the esophagogastrointestinal tract. In: Stem Cells. Regenerative Medicine and Cancer. Singh SR (ed.), Nova Science Publishers, Inc, New York, USA. pp. 279-308, 2010.

16 Baker AM, Cereser B, Melton S, Fletcher AG, Rodriguez-Justo M, Tadrous PJ, Humphries A, Elia G, McDonald SA, Wright NA, Simons BD, Jansen M and Graham TA: Quantification of crypt and stem cell evolution in the normal and neoplastic human colon. Cell Rep 8: 940-947, 2014.

17 Kang H and Shibata D: Direct measurements of human colon crypt stem cell niche genetic fidelity: The role of chance in nonDarwinian mutation selection. Front Oncol 3: 264-269, 2013.
Received June 19, 2018

Revised June 25, 2018

Accepted June 28, 2018 